\title{
UN MODELO EXPLICATIVO DE LA CALIDAD EMPRESARIAL EN ANDALUCÍA
}

Joaquín GUZMÁN CUEVAS *

Jesús BASULTO SANTOS*

Carlos ARIAS MARTÍN *

Francisco Javier SANTOS CUMPLIDO *

\section{INTRODUCCIÓN.}

Desde una perspectiva estrictamente económica, los estudios de la calidar empresarial suelen referirse a la cuenta de resultados de una determinada empres: o sector productivo. Como consecuencia de ello, los análisis que se desarrolla en este sentido, tanto teóricos como empíricos, suelen centrar su atención en la distintas parcelas que componen la gestión empresarial (finanzas, administración organización, marketing, etc.), es decir, estos estudios se centran en la esferi gerencial del empresario. Sin embargo, en el mundo empresarial y, especialments en su sector más numeroso, la pequeña y mediana empresa, el nivel de calidar no solamente depende de una certera gestión, sino también de una serie $d \mathfrak{c}$ cualidades que caracterizan al buen empresario respecto a otros colectivos de $l_{i}$ población activa. En este segundo caso, nos estamos refiriendo a las cualidade: que explican la función promotora o dinamizadora de nuevos proyectos, aspectc si cabe más relevante para la actividad de la empresa que la propia gestión.

El contenido de este trabajo se sitúa esencialmente en esta última vertiente en las cualidades del empresario. Obviamente, las cualidades que caracterizan a buen empresario no están suficientemente bien definidas y delimitadas. Nc obstante, sobre la amplia base empírica que proporcionan más de trescienta: entrevistas realizadas a empresarios de la provincia de Sevilla, este trabajo intentc una aproximación a un indicador representativo de la calidad del empresario c

*. Departamento de Económica Aplicada I. de la Universidad de Sevilla. 
partir de cuatro rasgos cualitativos unánimemente reconocidos de entre los que pueden configurar el espíritu empresarial.

\section{ELEMENTOS CUALITATIVOS CONSIDERADOS.}

En efecto, los aspectos cualitativos que caracterizan la actividad empresarial, son numerosos y diversos. Pero, independientemente del origen interno o externo a la propia persona o de la naturaleza innata o adquirida de tales aspectos, podemos citar los siguientes atributos empresariales: la iniciativa, la creatividad, la ambición, la adicción al trabajo, la independencia, la imaginación o el control interno. No obstante, desde una perspectiva empírica, podemos señalar cuatro elementos característicos que discriminan con nitidez la calidad del empresario y que nos sirven para medir algunos de los atributos anteriormente citados.

a) Preferencia por el trabajo por cuenta propia.

Dentro de unos mismos niveles de ingresos, la inclinación por eI autoempleo frente al trabajo por cuenta ajena, constituye un rasgo destacado de la personalidad del empresario, puesto que, de esta forma, se determina su deseo de independencia. No obstante, la preferencia por trabajar por cuenta propia no representa una condición suficiente pero si necesaria para un mínimo nivel de rendimiento empresarial pues es necesario completar el deseo de independencia con otros rasgos.

b) Motivación vocacional.

Lógicamente, como en toda actividad profesional, los niveles de rendimiento y por consiguiente las probabilidades de éxito, son mayores cuando subyace un motivo vocacional que en el caso en que se desarrolla una actitud por otras razones como la maximización de beneficios o la supervivencia económica. En este sentido, al igual que identificamos anteriormente la preferencia a trabajar por cuenta propia con el deseo de independencia del empresario, la motivación vocacional podría ser, en principio, identificada entre los distintos atributos empresariales mencionados anteriormente con la adición al trabajo. No obstante, es preciso señalar que la motivación debe entenderse, ya sea vocacional o de cualquier otro tipo, 
como resultado de los factores psicológicos, sociológicos o económicos que actúan sobre el comportamiento del empresario, induciéndole a unć determinada actitud.

c) Apoyo técnico de gestión.

En la esfera gerencial o "management" se dan diversas facetas (inversora comercial, etc.). Pero la especialización en estas funciones solo adquiere sentido en las empresas de cierta dimensión, mientras que en las pequeñas empresas y microempresas el hombre-empresario es el que suele asumit del mejor modo posible cada una de estas facetas de gestión. Contar al menos con los servicios de un experto contable-financiero o de algún gabinete de asesoramiento más allá de la cumplimentación de las obligaciones legales (seguros sociales, IVA, etc.), constituye un exponente representativo de una mínima calidad de gestión técnica empresarial.

d) Petición de ayudas financieras.

La búsqueda de financiación constituye con gran diferencia el principal problema para los pequeños y medianos empresarios, según ellos mismos. Para paliarlos al menos en alguna medida, existe una amplia gama de programas de apoyos financieros a las PYMEs, tanto por parte de las distintas administraciones (comunitaria, nacional, regional y local), como por parte de entidades financieras (cajas de ahorro, banca oficial, banca pública, etc.). Los pequeños y medianos empresarios que han solicitado en alguna ocasión estas ayudas demuestran un claro síntoma de inquietud por intentar resolver sus propios problemas y, por consiguiente, iniciativa, control interno o ambición.

\section{ANÁLISIS DESCRIPTIVO DE LA MUESTRA.}

A continuación vamos a pasar a describir la muestra que se realizó para analizar la calidad de los empresarios de la provincia de Sevilla en base a las cuatro características que acabamos de definir. En primer lugar, es preciso hacer referencia a la inexistencia en el momento de elaborar la muestra de algún tipo de censo, no ya de empresarios, que es el colectivo objeto de estudio, sino de 
empresas. Para salvar este problema se tuvo que recurrir a distintas fuentes, como la Cámara Oficial de Comercio, Industria y Navegación de Sevilla, los listados de cotizaciones de la Seguridad Social, y las Confederaciones de Empresarios. Después de cruzar esta información, se construyó una lista de empresas que podrían ser de interés, y tras la correspondiente comprobación y revisión de las encuestas, fueron aceptadas un total de 308 , por lo que la muestra está formada por 308 empresarios residentes en Sevilla con al menos un trabajador a su cargo.

El análisis descriptivo de la muestra para cada una de las características de la calidad empresarial definidas anteriormente, nos conduce, en principio, a los siguientes resultados:

- El $60 \%$ de los encuestados no preferirían un trabajo estable con sueldo fijo que les permitiera mantener su nivel de vida.

- El 58\% considera que el principal motivo que les condujo a convertirse en empresarios fue la vocación.

- El 71\% cuenta con algún experto contable o mantienen relación con algún gabinete asesor.

- El 40\% ha solicitado en alguna ocasión financiación especial.

Ahora bien, a partir de las distribuciones de frecuencias condicionadas, podemos comprobar que estos porcentajes se van incrementando, aunque, obviamente, su frecuencia absoluta disminuye. Así, si partimos de aquellos que no quieren un trabajo estable, el $69 \%$ se inclina por una motivación vocacional, frente al $58 \%$ anterior; si a continuación consideramos tanto aquellos que no prefieren un trabajo estable y además tienen una motivación vocacional, el porcentaje de los que cuentan con algún experto contable o mantiene relación con algún gabinete asesor se eleva al 79\%; y, por último, el tanto por ciento de aquellos que han solicitado alguna vez financiación especial, y que también no prefieren un trabajo estable, tienen una motivación vocacional, y mantienen relación con algún experto contable o con algún gabinete asesor, asciende al $45 \%$.

Por todo ello, se tiene un conjunto de 46 empresarios, lo que representa aproximadamente el $15 \%$ de los encuestados, que han manifestado poseer las cuatro características que denotan la calidad empresarial. Las características de clasificación las componen cinco variables categónicas. La distribución de frecuencias de estas variables son las que se ofrecen a continuación: 
Formula empresarial Individual

Familiar

Sociedad no familiar

$\underline{\text { Sector }}$

Hostelería

Comercio

Otros servicios

Industria y construcción
Frecuencia

182

75

51

60

137

59

52

55

195

58

187

121
Porcentaje

59,1

24,4

16,5

19,5

44,5

19,2

16,8

17,9

63,3

18,8

60,7

39,3

Edad con que se hizo empresario

Menos de 26 años

113

De 26 a 30 años

120

36,7

Más de 30 años

75

24,3

\section{MODELIZACIÓN DE LAS CARACTERÍSTICAS DE LA CALIDAD EMPRESARIAL.}

Para modelizar cada una de las cuatro características que conforman la calidad empresarial, hemos acudido a la regresión logística, y así, en general, si Y es una variable aleatoria dicotómica, y si $\mathrm{X}_{1}, \ldots, \mathrm{X}_{\mathrm{k}}$ es un conjunto de variables aleatorias, se puede estimar la probabilidad de que $\mathrm{Y}=\mathrm{I}$, que viene dada por:

$$
P(Y=1 / Z)=\frac{e^{z}}{1+e^{z}}=\frac{1}{1+e^{-z}}
$$

donde $\mathrm{Z}$ es la combinación lineal:

$$
\mathrm{Z}=\beta_{0}+\beta_{1} \cdot \mathrm{X}_{1}+\ldots+\beta_{\mathrm{k}} \cdot \mathrm{X}_{\mathrm{k}}
$$


y donde:

$$
\mathrm{P}(\mathrm{Y}=0 / \mathrm{Z})=1-\mathrm{P}(\mathrm{Y}=1 / \mathrm{Z})
$$

Una vez estimado los coeficientes $\beta_{\mathrm{i}}(\mathrm{i}=0,1, \ldots, \mathrm{k})$, mediante el método de la máxima verosimilitud, se podrá estimar $\mathrm{P}(\mathrm{Y}=1 / \mathrm{Z})$. Ahora bien, para interpretar adecuadamente estos coeficientes, debemos resaltar que si $X_{i}$ es una variable cuantitativa, la razón:

$$
C=\frac{P(Y=1 / Z)}{P(Y=0 / Z)}=e^{\beta_{0}} \cdot e^{\beta_{1} \cdot X_{1}} \ldots e^{\beta_{k} \cdot X_{k}}
$$

$y$ de esta forma cuando $X_{i}$ se incrementa, por ejemplo de 0 a 1 , se tiene:

a) Si $\beta_{i}=0$, el valor de $C$ no se altera.

b) Si $\beta_{i}>0$, el valor de $C$ se incrementa en $e^{\beta i}$.

c) Si $\beta_{\mathrm{i}}<0$, el valor de $C$ se decrementa en $\mathrm{e}^{\beta \mathrm{i}}$.

De esta forma, conforme mayor sea el valor de $\mathrm{e}^{\beta \mathrm{i}}$, mayor será la variación producida en la estimación de $\mathrm{P}(\mathrm{Y}=1 / \mathrm{Z})$ ante una alteración en $\mathrm{X}_{\mathrm{i}}$.

Por otra parte si $\mathrm{X}_{\mathrm{i}}$ es una variable categórica, para resolver el problema de la asignación numérica de las categorías, se puede considerar el siguiente planteamiento. Sea $X_{\mathrm{i}}$ una variable con tres categorías.

$$
\begin{aligned}
& \left\{\begin{array}{l}
X_{11}=1 \text { si toma la categoría } X_{1}=1 \\
X_{11}=0 \text { si no toma la categoría } X_{1}=1
\end{array}\right. \\
& \left\{\begin{array}{l}
X_{12}=1 \text { si toma la categoría } X_{1}=2 \\
X_{12}=0 \text { si no toma la categoría } X_{1}=2
\end{array}\right. \\
& \left\{\begin{array}{l}
X_{13}=0 \text { en cualquier caso }
\end{array}\right.
\end{aligned}
$$


De esta forma, la combinación lineal original:

$$
Z=\beta_{0}+\beta_{1} \cdot X_{1}+\ldots+\beta_{k} \cdot X_{k}
$$

se transforma en:

$$
Z=\beta_{0}+\beta_{11} \cdot X_{11}+\beta_{12} \cdot X_{12}+\ldots+\beta_{k} \cdot X_{k}
$$

y entonces, si consideramos la razón

$$
C=\frac{P(Y=1 / Z)}{P(Y=0 / Z)}=e^{\beta_{0}} \cdot e^{\beta_{11} \cdot X_{11}} \cdot e^{\beta_{12} \cdot X_{12}} \cdot e^{\beta_{2} \cdot X_{2}} \cdot \ldots \cdot e^{\beta_{k} \cdot X_{k}}
$$

se puede comprobar que si $\mathrm{X}_{\mathrm{i}}$ toma la categoría 1 , el valor de la razón será:

$$
C_{1}=e^{\beta_{0}} \cdot e^{\beta_{11} \cdot X_{11}} \cdot e^{\beta_{2} \cdot X_{2}} \cdot \ldots \cdot e^{\beta_{k} \cdot X_{k}}
$$

mientras que si toma la categoría 2 , se transforma en:

$$
C_{2}=e^{\beta_{0}} \cdot e^{\beta_{12} \cdot X_{12}} \cdot e^{\beta_{2} \cdot X_{2}} \cdot \ldots \cdot e^{\beta_{k} \cdot X_{k}}
$$

$\mathrm{y}$, por último, si consideramos que $\mathrm{X}_{1}=3$, es decir, toma la tercera categoría, se transforma en:

$$
C_{3}=e^{\beta_{0}} \cdot e^{\beta_{2} \cdot X_{2}} \cdot \ldots \cdot e^{\beta_{k} \cdot X_{k}}
$$

Por ello, los coeficientes $\beta_{11}$ y $\beta_{12}$ ofrecen la siguiente interpretación. Si $X_{1}$ toma la primera categoría, el valor de la razón $\mathrm{C}$ es precisamente $\mathrm{C}_{1}$, donde se verifica la relación $C=C_{1}=e^{\beta_{11}} C_{3}$. De esta forma, el coeficiente $\beta_{11}$ está comparado el efecto de la primera categoría de la variable respecto de la tercera categoría 
de la misma, que se toma como patrón. Entonces, si $\beta_{11}$ es positivo, tenemos que $\mathrm{C}_{\mathrm{I}}>\mathrm{C}_{3}$; es decir, que $\mathrm{P}(\mathrm{Y}=1 / \mathrm{Z})$ cuando $\mathrm{X}_{1}$ toma la primera categoría es superior a $P(Y=1 / Z)$ cuando $X_{1}$ toma la tercera categoría; cuantificándose la diferencia entre los valores de $C_{1} y$ de $C_{3}$ por el valor de $e^{\beta_{1}}$. Es decir, si $X_{1}$ toma la primera categoría, tenemos que $C=C_{1}=e^{\beta_{11}} C_{3}$, y por lo tanto, si por ejemplo, $\beta_{11}$ es positivo, el valor de $\mathrm{e}^{\beta_{11}}$ ofrece el incremento de la razón $\mathrm{C}$ que se produce cuando $X_{1}$ toma la categoría 1 en lugar de la 3 . Por lo tanto, los valores de $e^{\beta_{11}}$ $y$ de $\mathrm{e}^{\beta_{12}}$ deben ser interpretados como la variación producida en $\mathrm{C}$ cuando en lugar de tomar $\mathrm{X}_{1}$ la categoría 3 toma la 1 y la 2 , respectivamente.

Evidentemente, en el caso de que $X_{1}$ sea una variable dicotómica, puede considerarse como una variable categórica con dos categorías, e interpretarse por tanto de la misma forma que se acaba de exponer. Para determinar la significatividad de los coeficientes $\beta_{\mathrm{i}}$ se puede emplear el estadístico de Wald, que para muestras grandes, tiene una distribución ji-cuadrado con un grado de libertad, pudiendo expresar el valor del estadístico, para el coeficiente $\beta_{\mathrm{i}}$ :

$$
W_{i}=\left(\frac{\hat{\beta}_{i}}{\sigma_{\hat{\beta}_{i}}}\right)^{2}
$$

donde $\beta_{\mathrm{i}}$ es el valor estimado de $\beta_{\mathrm{i}}$ y $\sigma_{\beta_{\mathrm{t}}}$ es su correspondiente desviación típica.

Por otra parte, dada la peculiar naturaleza de la variable dependiente, de los distintos indicadores que muestran la bondad del ajuste, se ha considerado el más simple, pero que a la vez puede ser el más ilustrativo, siendo éste el porcentaje de asignación correcta de $\hat{\mathrm{Y}}$, donde:

$$
\hat{\mathrm{Y}}=1 \text { si } \hat{\mathrm{P}}(\mathrm{Y}=1 / \mathrm{Z}) \geq 0,5
$$

y:

$$
\hat{\mathrm{Y}}=0 \text { si } \hat{\mathrm{P}}(\mathrm{Y}=1 / \mathrm{Z})<0,5
$$

De esta forma, dado que se dispone para cada observación del verdadero valor de $\mathrm{Y}$, y de su correspondiente valor estimado, $\hat{\mathrm{Y}}$ se podrá determinar el número de veces, que posteriormente debe ser expresado en porcentaje, en los que el 
valor de $Y$ coincide con su estimación, es decir, cuando $Y=0$ e $\hat{Y}=0$, o cuandc $\hat{Y}=1$, pues en el resto de los casos la estimación no es correcta.

Los resultados obtenidos al realizar la regresión logística para cada una de las cuatro variables que conforman la calidad empresarial son los siguientes: (Ver Tabla I)

Las estimaciones señaladas con * han resultado significativas para un nive] de significación del 5\%; las categorías que no aparecen en la tabla, y que precisamente son las que sirven de referencia son las siguientes: sociedades nc familiares para la fórmula empresarial, industria y construcción para el sector de actividad, más de seis trabajadores para el número de trabajadores, y más de $3 C$ años para la edad con que el encuestado se hizo empresario.

Debemos destacar la falta de significatividad que se aprecian en las variables para algunas de las características estudiadas. Así, por ejemplo, cuando sє considera la preferencia por el trabajo por cuenta propia, sólo la edad con la que el entrevistado se hace empresario es significativa, y por lo tanto, únicamente podemos señalar que son aquellos que se constituyeron en empresarios en sc edad más temprana, los que significativamente prefieren un trabajo por cuent $\varepsilon$ propia frente a otro por cuenta ajena que le permitiera mantener su nivel de vida

Por lo que respecta al motivo que le indujo a convertirse en empresario. podemos destacar que son los que su empresa pertenece a los sectores de: comercio y otros servicios, aquellos cuya empresa está ubicada en el áré metropolitana de Sevilla y los que se convirtieron en empresarios en una edac más tardía, los que menor motivación vocacional presentan.

Si consideramos a continuación la característica de contar con algún expertc contable o de mantener relaciones con algún gabinete asesor, podemos señalar que, por un lado, son las empresas individuales las que en menor medide presentan esta característica, y por otro, tal y como era de prever, que las empresas con menor número de trabajadores son las que también, en menor medida, cuentan con apoyo técnico de gestión.

Por último, y por lo que respecta a la solicitud de ayudas financieras, únicamente no se aprecian diferencias significativas en la variable número de trabajadores. De esta forma, podemos señalar que son las empresas constituidas er cualquier tipo de sociedad no familiar, las pertenecientes al sector del comercio y las ubicadas en el área metropolitana de Sevilla, las que en menor medida har solicitado en alguna ocasión financiación especial; mientras que son los entrevis. tados que se hicieron empresarios con más de treinta años, los que en mayos medida han realizado alguna vez esta petición. 


\begin{tabular}{|c|c|c|c|c|c|c|c|c|}
\hline & \multicolumn{2}{|c|}{$\begin{array}{l}\text { Trabajo por } \\
\text { cuenta propia }\end{array}$} & \multicolumn{2}{|c|}{$\begin{array}{l}\text { Motivación } \\
\text { vocacional }\end{array}$} & \multicolumn{2}{|c|}{$\begin{array}{l}\text { Apoyo técnico } \\
\text { de gestión }\end{array}$} & \multicolumn{2}{|c|}{$\begin{array}{l}\text { Petición de ayudas } \\
\text { financieras }\end{array}$} \\
\hline & $\beta_{\mathrm{i}}$ & $\exp \left(\beta_{\mathrm{i}}\right)$ & $\beta_{\mathrm{i}}$ & $\exp \left(\beta_{1}\right)$ & $\beta_{\mathrm{i}}$ & $\exp \left(\beta_{i}\right)$ & $\beta_{\mathrm{i}}$ & $\exp \left(\beta_{\mathrm{i}}\right)$ \\
\hline \multicolumn{9}{|l|}{ Fórmula } \\
\hline Individual & $-0,2061$ & 0,8137 & $-0,1107$ & 0,8952 & $-1,2970 *$ & 0,2734 & $-1,1294 *$ & 0,3233 \\
\hline Familiar & 0,3831 & 1,4668 & $-0,1932$ & 0,8243 & $-0,9256$ & 0,3963 & $-0,9300 *$ & 0,3946 \\
\hline \multicolumn{9}{|l|}{ Sector } \\
\hline Hostelería & $-0,2896$ & 0,7486 & $-0,4364$ & 0,6463 & 0,0443 & 1,0452 & 0,1020 & 1,1074 \\
\hline Comercio & $-0,6173$ & 0,5394 & $-0,9009 *$ & 0,4062 & $-0,3651$ & 0,6941 & $-0,8218 *$ & 0,4397 \\
\hline Otros servicios & 0,0061 & 1,0061 & $-0,9976^{*}$ & 0,3688 & $-0,6614$ & 0,5161 & $-0,4235$ & 0,6548 \\
\hline \multicolumn{9}{|l|}{$\mathrm{N}^{\circ}$ trabajadores } \\
\hline Uno & $-0,8188$ & 0,4409 & $-0,4954$ & 0,6093 & $-1,3877 *$ & 0,2497 & 0,0821 & 1,0856 \\
\hline Dos-seis & $-0,0245$ & 0,9758 & 0,3204 & 1,3777 & $-0,7284$ & 0,4827 & 0,1934 & 1,2133 \\
\hline \multicolumn{9}{|l|}{ Localidad } \\
\hline A.Metropolitana & 0,0764 & 1,0794 & $-0,8591 *$ & 0,4236 & $-0,3102$ & 0,7333 & $-2,0214^{*}$ & 0,1325 \\
\hline \multicolumn{9}{|l|}{ Edad } \\
\hline Menos de 25 años & $0,6074 *$ & 1,8357 & $0,7826^{*}$ & 2,1871 & 0,0896 & 1,0938 & $-0,9270 *$ & 0,3957 \\
\hline De 26 a 30 años & 0,2260 & 1,2536 & $0,6568 *$ & 1,9285 & $-0,1044$ & 0,9009 & $-0,7100^{*}$ & 0,4917 \\
\hline Constante & 0,6084 & & $0,9933 *$ & & $3,2793 *$ & & $2,5498 *$ & \\
\hline
\end{tabular}


Por lo que respecta al porcentaje de asignación correcta para cada una de las cuatro características que denotan la calidad empresarial, podemos señalar que es el $64,43 \%$ para el trabajo por cuenta propia, el $67,45 \%$ para la motivación vocacional, el $73,49 \%$ para el apoyo técnico de gestión, y el $68,12 \%$ para la petición de ayudas financieras.

\section{CONSTRUCCIÓN DE UN INDICADOR ÚNICO DE LA CALIDAD EMPRESARIAL.}

Basándonos en las cuatro características que conforman la calidad empresarial, podríamos formar 5 grupos ente los encuestados. El primero estaría formado por aquellos que no poseen ninguna de las cuatro características; es decir, aquellos que prefieren un trabajo estable, que su motivación no es vocacional, que no tienen apoyo técnico de gestión y que, por último, tampoco han solicitado alguna vez financiación especial (Grupo 1). El segundo grupo estaría formado por aquellos que han manifestado poseer una de estas características (Grupo 2), el tercero por los que tienen dos de ellas (Grupo 3), el cuarto por los que poseen tres de las cuatro (Grupo 4), y el último grupo lo formarían los entrevistados que tienen todas las características (Grupo 5). Se observa que a medida que nos movemos hacia grupos de mayor valor numérico, aumenta el número de categorías positivas de las variables dicotómicas bajo estudio. De esta forma, los grupos así creados definen un indicador de calidad empresarial en una escala ordinal.

Ahora bien, si pretendemos obtener un indicador ordinal que mida la calidad empresarial, y que tenga en cuenta las diferencias entre las cuatro variables dicotómicas que se consideran, se puede proponer un análisis de correspondencias múltiples. De esta forma, podemos construir una nueva variable, combinación lineal de las variables originales, que maximiza la suma de las razones de las correlaciones ente cada una de las variables originales y la nueva variable. Una vez obtenida esta nueva variable, se puede observar que únicamente admite un total de 16 valores distintos, que junto a los valores de las variables originales se presentan en la tabla II.

Tal y como se puede observar en la tabla, la nueva variable crea diferencias entre los encuestados, pues de aquellos que sólo poseen una de las cuatro características que denotan la calidad empresarial, asigna mayor puntuación a los que manifiestan tener apoyo técnico de gestión; a continuación a los que se inclinaron únicamente por la motivación vocacional, posteriormente a aquellos que de las cuatro características sólo manifestaron haber solicitado alguna vez ayudas financieras, y por último, la característica menos importante se considera 
que es la preferencia por el trabajo por cuenta propia. El mismo razonamiento puede extenderse cuando el encuestado únicamente manifiesta que posee dos o tres de las características que denotan la calidad empresarial. Así, por ejemplo, cuando sólo se presentan tres de las cuatro características, la nueva variable asigna mayor puntuación a los que han manifestado tener apoyo técnico de gestión, motivación vocacional y haber solicitado alguna vez ayuda financiera.

\section{Tabla II}

\begin{tabular}{ccccc}
$\begin{array}{c}\text { Trabajo por } \\
\text { cuenta }\end{array}$ & $\begin{array}{c}\text { Motivación } \\
\text { vocacional }\end{array}$ & $\begin{array}{c}\text { Apoyo técnico } \\
\text { de gestión }\end{array}$ & $\begin{array}{c}\text { Petición de } \\
\text { ayudas financieras }\end{array}$ & $\begin{array}{c}\text { Nueva } \\
\text { Variable }\end{array}$ \\
\hline 0 & 0 & 0 & 0 & $-1,95$ \\
1 & 0 & 0 & 0 & $-1,45$ \\
0 & 0 & 0 & 1 & $-1,14$ \\
0 & 1 & 0 & 0 & $-0,97$ \\
0 & 0 & 1 & 0 & $-0,88$ \\
1 & 0 & 0 & 1 & $-0,65$ \\
1 & 1 & 0 & 0 & $-0,48$ \\
1 & 0 & 1 & 0 & $-0,39$ \\
0 & 1 & 0 & 1 & $-0,17$ \\
0 & 0 & 1 & 1 & $-0,08$ \\
0 & 1 & 1 & 0 & 0,09 \\
1 & 1 & 0 & 1 & 0,33 \\
1 & 0 & 1 & 1 & 0,42 \\
1 & 1 & 1 & 0 & 0,59 \\
0 & 1 & 1 & 1 & 0,90 \\
1 & 1 & 1 & 1 & 1,39
\end{tabular}

Así pues, una vez construido el indicador único de la calidad empresarial debemos abordar el estudio de este indicador mediante las características de clasificación contempladas anteriormente (fórmula empresarial, sector, $\mathrm{n}^{\mathrm{o}}$ de trabajadores, localidad, edad con que se hizo empresario). En este sentido, si cuando se consideró por separado cada una de las cuatro características que conforman la calidad empresarial, la significación de las variables no era la más idónea, parece razonable pensar que para este supuesto el poder explicativo de las mismas no alcance unos niveles muy altos. 
En efecto se ha realizado un análisis discriminante a los dieciséis grupos que aparecen en la tabla anterior, resultando que el porcentaje de asignación correcta de los encuestados es el $24,35 \%$. Una forma de aumentar este porcentaje de asignación es reducir el número de grupos; y así, considerando únicamente los cinco grupos a los que se alude en el comienzo de este epígrafe, se tienen los siguientes resultados:

\section{Tabla III}

\begin{tabular}{|c|c|c|r|r|r|c|}
\cline { 3 - 7 } \multicolumn{2}{c|}{} & \multicolumn{5}{|c|}{ PREDICCIÓN } \\
\hline Grupo & Frec & A & B & C & D & E \\
1 & 24 & $12(50,0 \%)$ & $4(16,7 \%)$ & $2(8,3 \%)$ & $3(12,5 \%)$ & $3(12,5 \%)$ \\
\hline 2 & 54 & $11(20,4 \%)$ & $14(25,9 \%)$ & $12(22,2 \%)$ & $11(20,4 \%)$ & $6(11,1 \%)$ \\
\hline 3 & 81 & $17(21,0 \%)$ & $8(9,9 \%)$ & $25(30,9 \%)$ & $11(13,6 \%)$ & $20(24,7 \%)$ \\
\hline 4 & 103 & $17(16,5 \%)$ & $11(10,7 \%)$ & $18(17,5 \%)$ & $31(30,1 \%)$ & $26(25,2 \%)$ \\
\hline 5 & 46 & $2(4,3 \%)$ & $4(8,7 \%)$ & $5(10,9 \%)$ & $10(21,7 \%)$ & $25(54,3 \%)$ \\
\hline
\end{tabular}

Los porcentajes que aparecen entre paréntesis son los de asignación correcta dentro de cada uno de los grupos, mientras que el global asciende al 34,7\%. Podemos destacar que en cualquiera de los grupos considerados el mayor porcentaje de asignación es siempre el del grupo adecuado, presentando sus valores más bajos precisamente para los tres grupos intermedios, siendo para los dos grupos extremos del $50 \%$ y $54,3 \%$.

Para valorar que categorías de las distintas variables son las que contribuyen a la discriminación de los grupos hemos calculado las correlaciones entre cada una de las categorías y la primera función discriminante. En el caso de que esta correlación sea negativa, querrá decir que son otras categorías de la misma variable las que tienen dependencia con el orden existente entre los grupos.

Las categorías que presentan mayores correlaciones en relación a la primera función discriminante, han sido:

$N^{o}$ de trabajadores:

Fórmula empresarial:

Localidad:

Localidad:
1

Individual

Otra

Área metropolitana de Sevilla
$(-0,58 \%)$

$(-0,51 \%)$

( $0,44 \%)$

$(-0,42 \%)$ 
Sector:

Industria y construcción

Fórmula empresarial:

Sociedad no familiar

Por ejemplo, para el caso de aquellos empresarios con un trabajador a su cargo (categoría primera de la variable número de trabajadores), el signo negativo de la correlación se interpreta diciendo que los empresarios que tienen un sólo trabajador tienden a ubicarse en los primeros grupos, es decir, en aquellos que sólo poseen una de las cuatro características que hemos seleccionado como explicativas de la calidad empresarial.

Si comparamos estos últimos resultados con los que se ofrecen en la regresión logística de cada uno de los indicadores que conforman la calidad empresarial, vemos que confirman de una forma global lo que se obtuvo para cada una de las variables dicotómicas.

\section{CONCLUSIONES.}

En este estudio, hemos analizado la calidad de los empresarios de la provincia de Sevilla en base a cuatro características que la definen. Para ello, se han utilizado diversas técnicas estadísticas como la regresión logística, el análisis de correspondencias múltiples o el análisis discriminante. Los resultados que se han obtenido nos demuestran lo siguiente:

a) Como habíamos expuesto anteriormente el deseo de trabajar por cuenta propia es una característica relevante del empresario pero no es determinante en su nivel de calidad. Como ha puesto de manifiesto el análisis de correspondencias múltiples es la que obtiene peores resultados de las cuatro características seleccionadas. No obstante, también es necesario reseñar, a partir de los resultados de la regresión logística, que aquellas personas que han decidido hacerse empresarios con menos de veinticinco años conceder gran importancia al trabajo por cuenta propia. Por tanto, si identificamos esta preferencia por el trabajo por cuenta propia con el deseo de independencia, son los más jóvenes los que más la valoran.

b) La tercera característica por orden de importancia según el análisis efectuado es la petición de ayuda financiera. Los problemas financieros son los más importantes para los empresarios, según su propia opinión, y, sin embargo, nos encontramos que los resultados obtenidos no valoran en gran medida la iniciativa que entraña resolverlos. No obstante, de los datos que nos proporciona la regresión 
logística, podemos definir el perfil de los empresarios que, en mayor medida realizan peticiones de ayudas financieras en base a las cinco variables categóricas: son personas que se convirtieron en empresarios con más de 30 años y sor titulares de empresas principalmente del sector industrial, que tienen carácter nc familiar, situadas fuera del área metropolitana de Sevilla. La variable número de trabajadores no resulta, en principio, significativa en la petición de ayuda financiera, según los resultados obtenidos, dada la pequeña dimensión media de la mayor parte de nuestros establecimientos. Por otra parte, es lógico que la actividad industrial, al exigir una mayor cantidad de recursos financieros por su mayor complejidad, induzca al empresario a pedir ayudas.

c) En cuanto a la motivación vocacional, aspecto que es resultado de la acción de diversos factores tales como psicológicos o sociológicos, el análisis estadístico la clasifica en segundo lugar en orden de importancia. Las motivaciones que inducen a los empresarios a ejercer su actividad son múltiples, sin embargo, la vocación es la más relevante porque las personas que la poseen, por regla general, cuando actúan no lo hacen pensando en la obtención del beneficic a corto plazo, sino en el desarrollo de la empresa a lo largo de sus vidas. Según la regresión logística, los empresarios que tienen esta vocación son personas que se han convertido en empresarios ante de los treinta años, resultado explicable porque los más jóvenes, debido a su carácter más independiente o a sus menores obligaciones, principalmente familiares, no se sienten motivados únicamente por cuestiones económicas. También, es destacable, como resultado de la regresión logística, que los empresarios con mayor vocación suelen pertenecer al sector industrial, aspecto lógico pues la actividad industrial es, por regla general, de mayor complejidad que la actividad de servicios, sobre todo, cuando se trata de servicios simples como comercios al por menor, que son los predominantes en Sevilla.

d) Por último, el análisis estadístico realizado nos dice que es el apoyo técnico de gestión la variable o característica que más incluye en la calidad del empresario. De las diferentes funciones que desempeña el empresario, la función gerencial aparece siempre como una de las más importantes. El carácter cada vez más sofisticado de la economía requiere para el empresario el esfuerzo de gestionar adecuadamente la empresa. En este sentido, la colaboración de un experto es la solución pues, hasta el momento, el nivel de formación del empresario es reducido. No obstante, también es cierto que el empresario debe dedicarse a realizar otro tipo de actividades en la empresa, que no sean las de administración. Fundamentalmente, podríamos decir que la actividad principal que debe 
ejercer el empresario es la de dinamización de la empresa ideando nuevos proyectos de inversión. En cuanto al valor explicativo de las variables categóricas sobre el apoyo técnico de gestión, la regresión logística nos expone que son las empresas no familiares y con más de seis trabajadores las más determinantes. Las pequeñas empresas del sector comercio, sin embargo, prefieren no contar con este tipo de apoyos pues no lo consideran necesario debido a la escasa complejidad de su actividad y al elevado coste que puede suponer.

En definitiva, estos aspectos son muy importantes cuando se analizan los problemas de nuestras empresas. Hasta el momento, los estudios sobre la calidad del empresario han sido muy escasos en España. Sin embargo, si queremos elaborar una adecuada política de apoyo a las empresas, especialmente a las PYMEs, no debemos olvidar en su diseño las medidas oportunas para mejorar la calidad del empresario que, en realidad, es quien dirige e impulsa a la empresa. En el caso de Sevilla, debido a la gran cantidad de microempresas y pequeñas empresas, fundamentalmente del comercio al por menor, la calidad de nuestros empresarios no es la más adecuada puesto que no demuestran una actitud muy positiva hacia las cuatro características que hemos tomado para medir la calidad empresarial. Por tanto, la labor que debe realizar la política económica sobre el empresario es más compleja en Sevilla que en otras zonas donde el tamaño medio de las PYMEs sea mayor y la actividad económica no esté dominada por el comercio al por menor.

\section{BIBLIOGRAFÍA.}

BINKS, M and VALES, P (1990): Entrepreneurship and Economic Change. McGraw Hill. London. BROOKSBANK, R (1991): Defining the Small Busines: a new classification of company size.

Entrepreneurship and Regional Development. 3.

CASSON, M. (1982): The Entrepreneur: An Economic Theory. Martin Robertson. Oxford.

COOPER, A.C. and DUNKELBERG, W.C. (1986): Entrepreneurship and Path to Business

Ownership. Strategic Management Journal. Vol 7, 53-68.

GUZMÁN, J. (1986): El sistema Financiero y las PYMEs Andaluzas. Instituto de Desarrollo Regional . Sevilla.

GUZMÁN, J. y PAZ, M. (1991): The Entrepreneurial Promotion in Depressed Areas: The Case

of Southern Spain. Ponencia presentada al 31st RSA European Congress. Lisboa.

JUIEN, P.A. (1989): The Entrepreneur and Economic Theory. International Small Bussines Journal. 7.3.

LACHENBRUCH, P.A. (1975): Discriminant Analysis. Hafner Press. New York.

McCULLAGH, P. and NELDR, J.A. (1983): Generalized Linear Models. Chapman \& Hall. London. VALLES, J., GUZMÁN, J. y PAZ, M. (1988): Hacia un Perfil Cualitativo del Empresario en Andalucía. Volumen homenaje al profesor García Barbancho. Consejería de Hacienda y Planificación. Junta de Andalucía. 